Helgoländer wiss. Meeresunters. 23, 459-466 (1972)

\title{
Zur Taxonomie und Ökologie mediterraner Holothuroidea-Apoda
}

\author{
L. v. SAlvini-Plawen \\ 1. Zoologisches Institut der Universität Wien; Wien I, Österreich
}

\begin{abstract}
On taxonomy and ecology of Mediterranean Holothuroidea-Apoda. Three species of apodous holothurians have been recorded from sea bottoms off Livorno (Italy). The findings contribute to our knowledge on the geographical distribution of Labidoplax buski (M'INTOSH) and Leptosynapta minuta (BeCHER) and lead to the description of the mesopsammobiotic Trobbodota furcipraedita $\mathrm{n}$. sp. The new species differs from related known species in regard to its simply forked tentacles, the size of its calcareous wheels, and the shape of its sigmoid spicules. Some apodous holothurians from the mid-Adriatic Sea could be identified as belonging to a new species, Myriotrochus geminiradiatus n. $\mathrm{sp}$. This first Mediterranean representative of the genus $M$ yriotrochus is characterized by its 10 tentacles without digits and the special structure of the wheels.
\end{abstract}

\section{EINLEITUNG}

Die Holothurien des Mittelmeeres haben mit der umfangreichen Studie von ToRTONESE (1965) eine nicht nur für den italienischen Bereich umfassende, sondern auch eine für das gesamte Mediterran grundlegende Darstellung erhalten. Es wird darin aber ebenso offensichtlich, daß die bekannten Formen fast ausschließlich der Makrofauna angehören, wogegen die Kleinformen von den bisherigen Forschungen weitgehend unbeachtet blieben. Speziell innerhalb der apoden Holothurien sind Arten mit geringer Körpergröße stark vertreten (vgl. CLARK 1907); so sind mit neueren Untersuchungen der Meiofauna auch fast ausschließlich Vertreter dieser Gruppe als neu gemeldet (vgl. Cherbonnier 1960, Cherbonnier \& Gulle 1968, Montcharmont 1968, Salvini-Plawen 1972a).

Im Zuge von Untersuchungen verschiedener Sedimentböden bei Livorno/Italien und in der Mittel-Adria konnten nun ebenfalls vier Arten apoder Klein-Holothurien aufgesammelt werden. Sie verdienen allein schon hinsichtlich der geographischen Verbreitung eine Mitteilung, und sie zeigen wiederum auf, daß die mediterrane Meiofauna noch weitgehend unbekannt ist (vgl. Monniot 1962, Salvini-Plawen 1972b, u. a.). So konnten im Rahmen dieser Studien zwei neue Holothurien-Species gefunden werden. Es handelt sich um die Arten Trochodota furcipraedita spec. nov. und Myriotrochus gerniniradiatus spec. nov., die nachfolgend beschrieben werden. 


\section{ERGEBNISSE UND DISKUSSION}

\section{Trocbodota furcipraedita spec. nov.}

Aus einem Grobsand der „Secche della Meloria " (Livorno) in 4-5 m Tiefe entstammen zwei Individuen (Abb. 4) einer mesopsammalen Holothurie, welche auf Grund der Merkmale als eine neue Art der Gattung Trocbodota Ludwig zuzuordnen sind. Die beiden schlanken, sich terminad gleichmäßig verjüngenden Individuen messen (lebend) $5 \mathrm{~mm}$ bzw. $3 \mathrm{~mm}$, und zeigen am schrägen Oralfeld $10 \mathrm{bzw}$. 9 Tentakeln. Diese sind durch eine basale "Schirmhaut" untereinander verbunden und laufen sehr charakteristisch in ein gabelförmiges Ende aus, so daß daher je Tentakel nur zwei „Finger" vorhanden sind. Der gesamte Körper besitzt im Leben eine rosa Färbung und ist semi-transparent.

Der auffallend schräg angeordnete Kalkring setzt sich aus zehn fast hantelförmigen Einzelstücken zusammen (Abb. 1B). Die verstreut liegenden Kalkkörper (Abb. 1,6) bestehen aus den für die Gattung typischen sechs-speichigen Rädern und aus Sigmoiden, welche beide Elemente jedoch vom Bekannten etwas abweichen. Die runden bis schwach
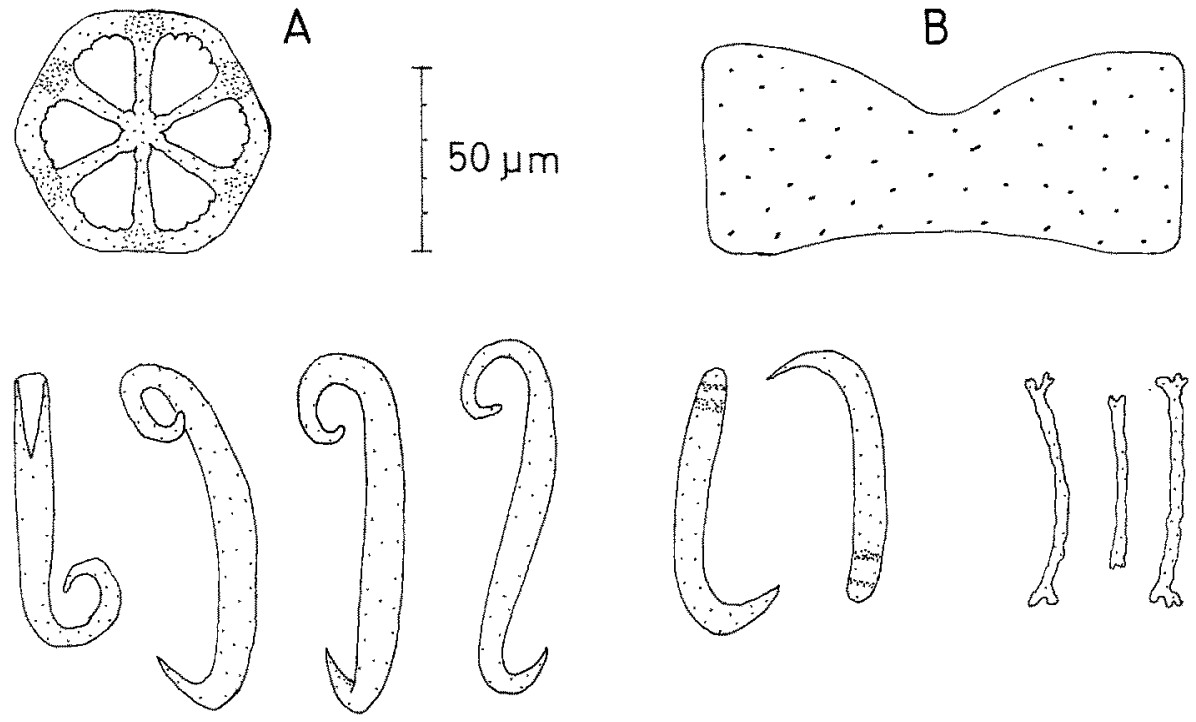

C

D

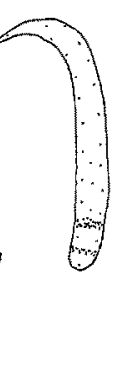

$E$

Abb. 1: Trochodota furcipraedita. A radförmiger Kalkkörper; $B$ ein Glied des Kalkringes; $C$ Sigmoide der Körperwand; $D$ Kalkkörper basal der Tentakel; $E$ Kalkkörper in den Tentakeln. (Der Maßstab gilt für alle Darstellungen)

sechs-kantigen Räder (Abb. 1 A) weisen einen Durchmesser von 40-70 $\mu \mathrm{m}$ auf und die Zähnelung am Innenrand ist nur in Andeutung mit 3-5 Dentikeln zwischen den Speichen manchmal sichtbar. Die sechs Speichen sind hierbei relativ schmal. Die Kalkkörper im Rumpf sind als typische Sigmoide ausgebildet und messen 70-90 $\mu \mathrm{m}$ (Abb. 
$1 \mathrm{C)}$. Das eine Ende ist hierbei jeweils hakenartig gekrümmt, wogegen das andere Ende mehr oder minder eingerollt ist; beide Endkrümmungen sind in ihrer Ebene meist um ca. $90^{\circ}$ versetzt. An den Tentakelbasen finden sich zudem einige abweichend gestaltete Körper vor (Abb. $1 \mathrm{D}$ ), welche nur an einem Ende gebogen sind und $60 \mu \mathrm{m}$ messen. Die zarten und schlanken Stäbe der Tentakel selbst (Abb. 1E) zeigen eine Länge von 55-60 $\mathrm{mm}$; ihr Körper ist etwas knotig strukturiert und die Enden laufen meist in $z$ wei gekerbte Gabel-Stïmpfe aus.

Beide vorliegenden Tiere sind nicht geschlechtsreif (Sammelzeit Juni). Thre innere Organisation läßt einen nahezu geraden Darm erkennen, welcher bei den fixierten Individuen eine orale Schleife bildet. Die für die Gattung charakteristische einzige Pol.xsche Blase ist deatlich erkennbar vorhanden. An den Basen dreier Tentakel konnte lateral je eine Statocyste festgestellt werden, deren Zahl wohl aber fünf oder zehn sein dürtte.

In systematischer Abgrenzung sind die vorliegenden Tiere innerhalb der Apoda auf Grund der sechs-speichigen Räder den Chiridotidae zuzuordnen, worunter sie hinsichtlich der Kalkstäbe den Sigmoinae angehören (vgl. Heding 1935 p. 7). Von den Gattungen dieser Gruppe zeigt Taeniogyrus SEMPER die Räder in Papillen angeordnet, wogegen verstreut angeordnete Räder für das Genus Trochodota LudwIG $(+$ Scoliodota ClarK) typisch sind (vgl. Clark 1907). Die vorliegenden Tiere gehören daher eindeutig zu Trochodota, für welche Gattung bisher neun Arten bekannt wurden (vgl. Clark 1907, Mortensen 1925, Hickmann 1962, Pawson 1968). Nur T. venusta (SEMON, 1887) und T. purpurea (LESSON, 1830) jedoch sind aus dem Mittelmeer resp. Südatlantik gemeldet, wogegen die sieben weiteren Arten allesamt dem west-pazifischen Raum angehören. Die vorliegende Art hebt sich jedoch einerseits durch die Radgröße und durch die einfach-gegabelten Tentakel von T. purpurea ab (bis $4 \mathrm{~cm}$; je 6-8 Tentakel-Finger; $\operatorname{Rad}-\phi 150-180 \mu \mathrm{m})$, und sie unterscheidet sich aber ebenso eindeutig von der geographisch nahen T. venusta. Besonders deren charakteristische Sigmoide, welche nur an einem Ende eingerollt sind, am anderen Ende hingegen einen glattwandigen Abschluß des keulig verdickten Stabes zeigen, sind neben den zehn-fingrigen Tentakeln hervorstechend-spezifische Merkmale (vgl. SEMoN 1887); T. venusta konnte ja seit dem Originalfund im Golf von Neapel erst in jüngster Zeit wieder gemeldet werden (Montcharmont 1968). Die vorliegenden Tiere stellen daher Vertreter einer gut abgegrenzten, neuen Art Trocbodota furcipraedita* dar.

Die Begleitfauna in der Fundprobe umfaßt durchwegs typische Mesopsammobionten. Neben Gastrotricha (2 Arten) und der Ascidie Psammostyela delamarei WeINSTEIN-MonNIot, sind vor allem Mollusken vertreten: Caecum glabrum (MoNTAGU), Microbedyle glandulifera (Kowalevsky), M.(?) milaschewitchii (Kowalevsky) und Philinoglossa (Philinoglossa) belgolandica Herturng.

Trochodota furcipraedita spec. nov.: Apode Holothurie mit zehn basal verbundenen und terminal einfach gegabelten Tentakeln; Kalkkörper als sechs-speichige Räder ( $\varnothing$ 40 $70 \mu \mathrm{m}$ ) und als beidendig gekrümmte Sigmoide (Abb. 1); Körpergröße (Jungtiere) bis über $5 \mathrm{~mm}$, Farbe rosa.

* furca $=$ Gabel oder gabelförmiges Gerät; praeditus = versehen mit; bezogen auf die Tentakel-Form. 


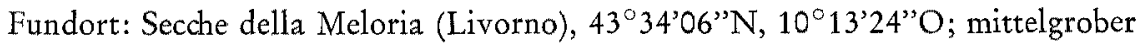
Schell-Sand, 4-5 m.

Der Typus ist unter der Nr. 13117 in der Evertebraten-Abteilung des Naturhistorischen Museums zu Wien deponiert.

Myriotrochus geminiradiatus spec. nov.

Die sechs Individuen der vorliegenden apoden Art aus der Adria wurden leider erst bei Untersuchung des fixierten Materials als von Labidoplax buski (vgl. p. 465) verschieden erkannt, so daß nur die wesentlichen Merkmale mitgeteilt werden können.

Die im Leben mit L. buski gleichfärbigen, also weißlichgrauen Tiere liegen mit Größen von $1 \mathrm{~mm}-2,3 \mathrm{~mm}$ vor und weisen allesamt zehn ungefingerte Tentakel auf; innerhalb der 'Tentakelspitze sind allerdings zwei (Sinnes-) Hödker auszunehmen (Abb. 2 B). Im Gegensatz zu L. buski sind keine Statocysten festzustellen.

Am mäßig gestreckten Körper selbst (Abb. 2 A) fällt die weitgehende Armut an Kalkkörpern auf, und an den vorliegenden Individuen konnten allein jeweils im hinteren Abschnitt 1-5 Räder festgestellt werden (die Tiere sind aber teils abgerissen). Diese Räder liegen in zwei Größen vor (Abb. $2 \mathrm{C}$ ), welche mit $\phi 35 \mu \mathrm{m} 15-16$ Speichen aufweisen, jene mit $\phi 45 \mu \mathrm{m}$ hingegen 20 Speichen besitzen. Beide Radgrößen gehören indes offenbar zum gleichen Typus, da der Aufbau übereinstimmt (Abb. 2 C). Hierbei muß hervorgehoben werden, daß keine horizontal ausgebildeten und zentripetal gerichteten Zähne bestehen, sondern daß die Elemente des Radringes je eine schräg zentripetal gerichtete Schuppe ausbilden (vgl. Abb. 2 D); die Anzahl der Radring-Elemente und der Schuppen stimmen zudem mit der Anzahl der Speichen überein, da die Speichen peripher in je ein entsprechendes Ring-Element übergehen. Die Speichen selbst bilden keine flügelartigen Ränder aus, sind aber demgegenüber paarig aufgebaut und stellen daher eigentlich Doppel-Speichen dar; sie sind in den größeren Rädern peripher sogar frei gegabelt (Abb. 2 C). Der Radteller erweist sich als gewölbt, so daß die Räder in Seitenansicht einem Hut gleichen (Sombrero, Abb. 2 D).

Der Kalkring besteht bei allen Individuen aus einem auffallenden Netzwerk von durchbrochenen Platten (Breite $70 \mu \mathrm{m}$; Abb. $2 \mathrm{E}$ ), so daß also wohl noch die Bildungsphase vorliegt. An den Tentakeln konnten keine Kalkkörper ausgenommen werden.

Trotz der Kenntnis der Organisation nur in einigen, allerdings wesentlichen Merkmalen, können die vorliegenden Individuen gut als eine eigene, neue Art abgegrenzt werden. Von den geographisch näheren, nord-europäischen Arten ( $M$. rinki STEENSTRuP $1851, M$. vitreus Sars 1866, $M$. theeli Ostergren 1905, M. eurycyclus Hedng 1935) sind die vorliegenden Tiere durch die Merkmalskombination von Tentakel und Radbau ebenso verschieden, wie von den pazifischen Vertretern ( $M$. minutus OstergreN 1905, M. mitsukurii OHSHIMA 1915, M. bathybius CLARK und M. giganteus CLARK 1920, M. brunni HANSEN 1956). Vielmehr erhebt sich eher die Frage, ob hiermit nicht Vertreter einer neuen Gattung oder eines Subgenus vorliegen, da der Radbau von M. geminiradiatus spec, nov." mit gleicher Schuppen- und Speichen-Zahl sich deutlich

$*$ geminus $=$ doppelt, paarig, Zwilling; radius $=$ Speiche. 
von jenen Rädern mit Zähnen in größerer Zahl abhebt. Wenn Trochoderma elegans THEel als eigene Gattung der Myriotrochidae bestehenbleibt (vgl. OST'ERGREN 1905), so wird bei einer zusammenfassenden Übersicht der Familie wohl auch für $M$. geminiradiatus zumindest ein eigenes Subgenus notwendig werden.
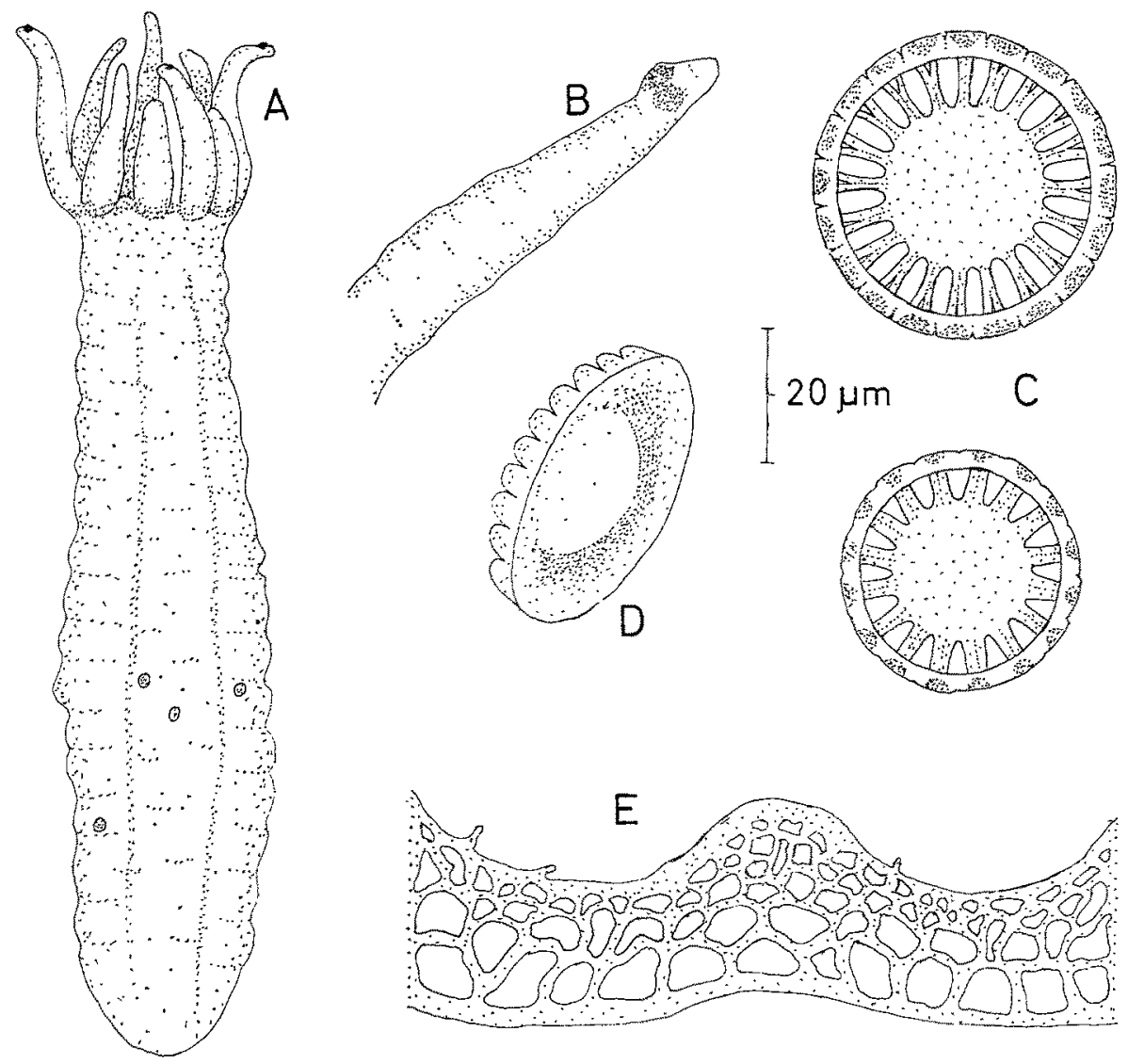

Abb. 2: Myriotrochus geminiradiatus. $A$ Habitus; $B$ ein Tentakel vergrößert; $C$ radförmige Kalkkörper in Aufsicht; $D$ radförmiger Kalkkörper in Schrägsicht; $E$ Ausschnitt aus dem Kalkring

Myriotrochus geminiradiatus spec. nov.: Apode Holothurie mit zehn ungefingerten T'entakeln; Kalkkörper allein aus wenigen Rädern bestehend ( $\varnothing 35-45 \mu \mathrm{m})$, deren peripher teils gespaltene, 15-20 Speichen doppelt angelegt sind und mit der Zahl der vorstehenden Schuppen (anstatt Zähnen) übereinstimmt; Körpergröße (Jungtiere) bis über $2,5 \mathrm{~mm}$, Farbe weißlichgrau.

Fundorte: Mittel-Adria, $42^{\circ} 25^{\prime} 06^{\prime \prime} \mathrm{N}, 15^{\circ} 20^{\prime} 36^{\prime \prime O}$; $150 \mathrm{~m}$, Schlamm mit Bryozoen; 4 Individuen. viduen.

Mittel-Adria, $43^{\circ} 23^{\prime} 00^{\prime \prime} \mathrm{N}, 1^{\circ} 08^{\prime} 12^{\prime \prime} \mathrm{O} ; 70 \mathrm{~m}$, graubrauner Schlamm; 2 Indi- 
Der Typus ist unter der Nr. 13118 in der Evertebraten-Abteilung des Naturhistorischen Museums zu Wien deponiert.
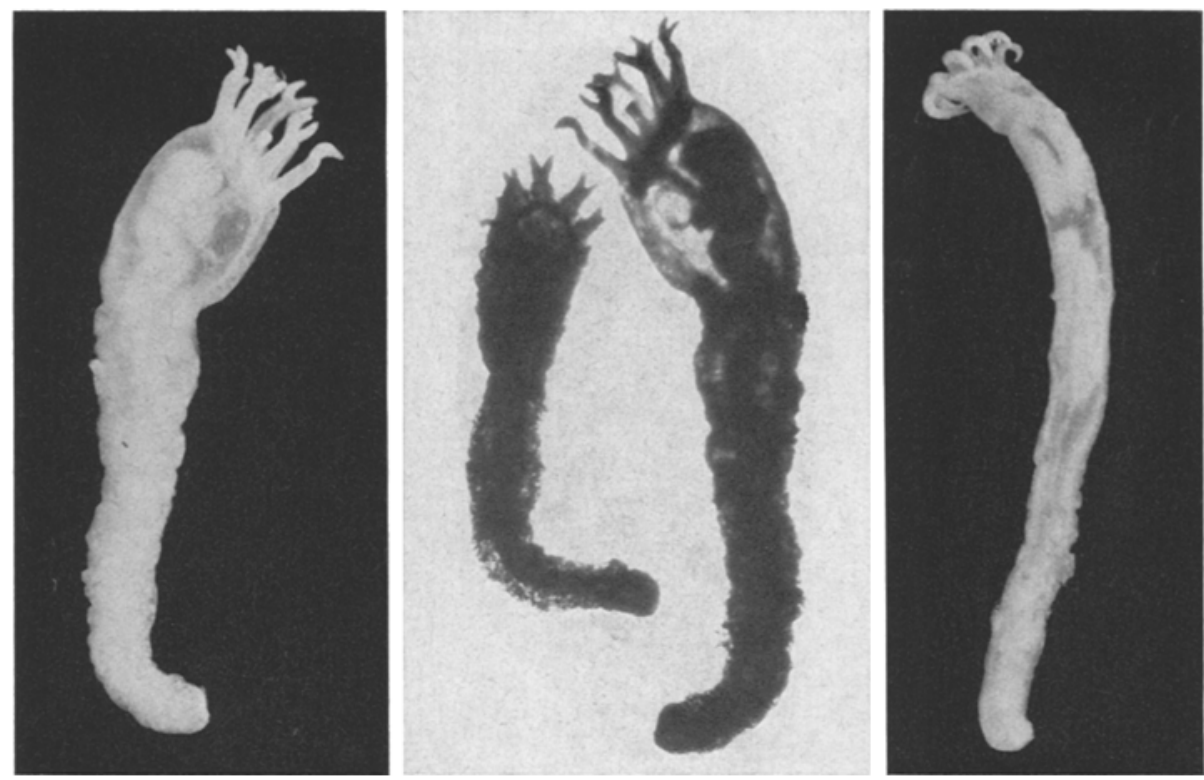

Abb. 3: Trodsodota furcipraedita, Typus

Abb. 4: Trochodota furcipraedita, Habitus beider Individuen Abb. 5: Leptosynapta minuta, Adulttier mit zwei Jungen

\section{Weitere Befunde}

Mit der vorgehend beschriebenen Trochodota furcipraedita konnte zu Rbabdomolgus ruber KEFERSTEIN aus der Nordsee und dem bretonischen Atlantik (vgl. Becher 1907), zu der indischen Psammothuria ganapatii Chandraserhara Rao (1968) und zu Leptosynapta minuta (BECHER 1906) die vierte mesopsammale Holothurie vorgestellt werden.

Für die letztgenannte Leptosynapta minuta können nun auch die "Secche della Meloria “ (4332'36”N, 1012'27”O; sauberer Grobsand, $15 \mathrm{~m}$ ) als neuer Fundort zweier Individuen hinzugefügt werden. Während TORTONESE (1965) in seiner Monographie auf diese Art als noch nicht gemeldet hinweisen muß, ist L. minuta nicht nur bei Marseille (Swedmark 1965, p. 94; Monniot 1962, p. 308), bei Banyuls-sur-Mer (Cherbonnier 1960) und bei Sète (Frze 1960) für das West-Mediterran nachgewiesen, sondern auch durch Montcharmont (1968) bei Neapel und nun ebenso bei Livorno für die Fauna Italiens aufzunehmen. Das größere der beiden vorliegenden Individuen (Abb. 5) war im Leben $7 \mathrm{~mm}$ lang und es enthält zwei schon völlig ausgebildete Jungtiere; das $\mathrm{z}$ weite Individuum ( $\mathrm{Abb}$. 7) ist kaum größer als jene und entstammt möglicherweise unmittelbar aus dem Adulttier. 
Eine weitere apode Klein-Holothurie, Labidoplax buski (M'INTOSH), war bis vor kurzem nur als auf den europäischen Nordatlantik beschränkt bekannt. PAwSON (1967) veröffentlichte den daher überraschenden Fund dieser Art vor der nordamerikanischen
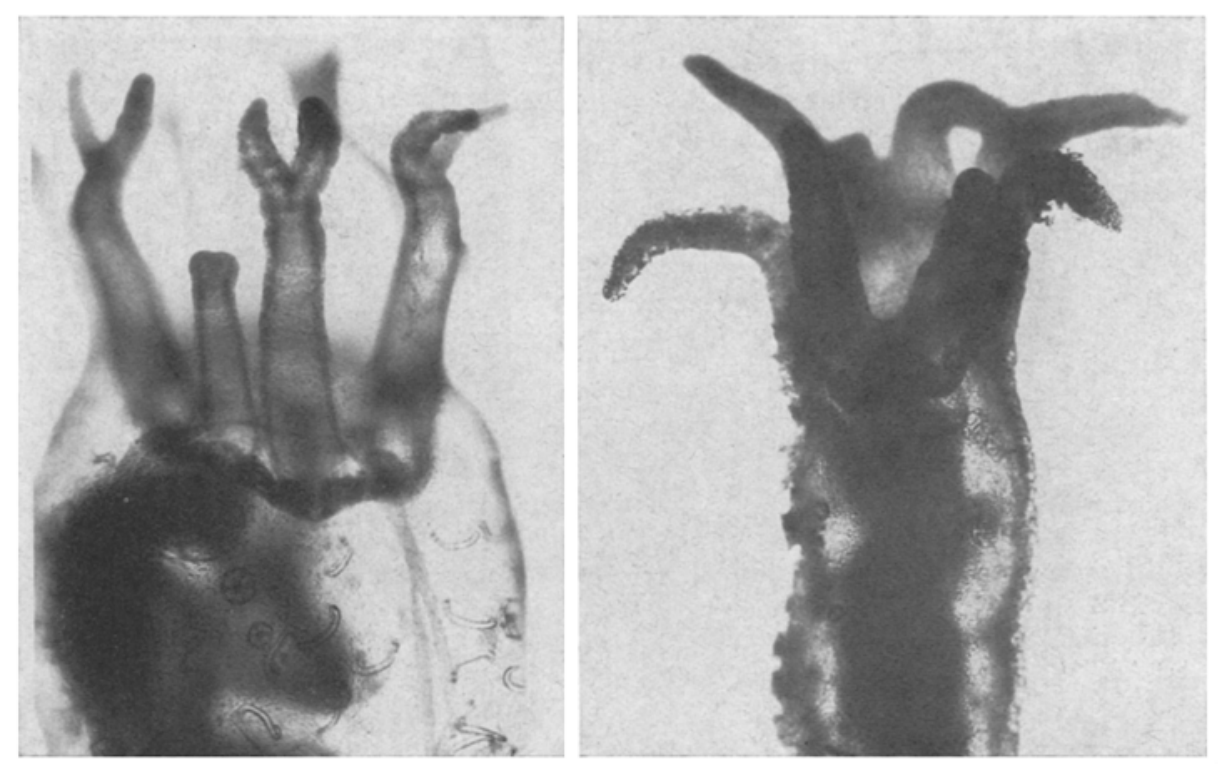

Abb. 6: Trochodota furcipraedita. Vorderende mit Rädern und Sigmoiden Abb. 7: Leptosynapta minuta. Vorderende des Jungtieres

Küste, erstmals außerhalb des genannten Bereiches. Schließlich geben CHERBonnier \& Guilde (1968) sowie Salvint-Plawen (1972a) die ersten mediterranen Funde bei Banyuls-sur-Mer bzw. aus der Adria bekannt. Labidoplax buski kann somit nunmehr für einen dritten Bereich im Mittelmeer gemeldet werden: Sie entstammt einem reinen Schlammboden aus $83-84 \mathrm{~m}$ unweit von Livorno $\left(43^{\circ} 28^{\prime} 18^{\prime \prime} \mathrm{N}, 10^{\circ} 08^{\prime} 24^{\prime \prime} \mathrm{O}\right)$; wie bei den Adria-Tieren sind als charakteristische Begleitfauna die Mollusca-Caudofoveata Prochaetoderma raduliferum und Falcidens gutturosus anzugeben (vgl. SALviNI-PLAWEN 1972b). Zudem können noch einige weitere Fundorte für L. buski in der Adria nachgetragen werden: $42^{\circ} 25^{\prime} 06^{\prime \prime} \mathrm{N}, 15^{\circ} 20^{\prime} 36^{\prime \prime} \mathrm{O} ; 150 \mathrm{~m} ; 2$ Individuen. $42^{\circ} 42^{\prime} \mathrm{N}$, $14^{\circ} 41^{\prime} \mathrm{O} ; 170 \mathrm{~m} ; 3$ Individuen. $42^{\circ} 32^{\prime} \mathrm{N}, 15^{\circ} 36^{\circ} \mathrm{O} ; 128 \mathrm{~m} ; 3$ Individuen. $43^{\circ} 23^{\prime} \mathrm{N}$, $14^{\circ} 08^{\prime} 12^{\prime \prime} \mathrm{O} ; 70 \mathrm{~m} ; 2$ Individuen. $43^{\circ} 38^{\prime} 31^{\prime \prime} \mathrm{N}, 15^{\circ} 47^{\prime} 48^{\prime \prime} \mathrm{O} ; 79 \mathrm{~m} ; 1$ Individuum. $43^{\circ} 44^{\prime} 36^{\prime \prime} \mathrm{N}, 15^{\circ} 42^{\prime} \mathrm{O} ; 71 \mathrm{~m} ; 1$ Individuum.

\section{ZUSAMMENFASSUNG}

1. Faunistische Untersuchungen an Sedimentböden vor Livorno (Italien) und in der Mittel-Adria erbrachten neue Kenntnisse an vier Arten apoder Klein-Holothurien.

2. Die mesopsammobionte Trochodota furcipraedita spec. nov. (Livorno) unterscheidet sich sowohl durch die einfach-gegabelten Tentakel, wie auch durch die radförmigen und sigmoiden Kalkkörper von den bisher bekannten Arten. 
3. Funde von Leptosynapta minuta bei Livorno und Labidoplax buski bei Livorno wie in der Adria erweitern die Kenntnis von der geographischen Verbreitung der Arten.

4. Myriotrochus geminiradiatus spec. nov. aus der Adria weist zehn ungefingerte Tentakel und Kalkräder von spezifischem Bau auf; mit dieser Art kann erstmalig auch die Gattung für das Mediterran nachgewiesen werden.

Danksagung. Die Untersuchungen wurden mit finanziellen Mitteln des „Fonds zur Förderung der wissenschaftichen Forschung in Osterreich" durchgeführt.

\section{ZITIERTE LITERATUR}

BECHER, S., 1906. Über Synapta minuta n. sp., eine brutpflegende Synaptide der Nordsee, und über die contractilen Rosetten der Holothurien. Zool. Anz. 30, 505-509.

- 1907. Rhabdomolgus ruber KePERstern und die Stammform der Holothurien. Z. wiss. Zool. 88, 545-689.

Chandraserhara Rao, G., 1968. On Psammotburia ganapatii n. gen. n. sp., an interstitial holothurian from the beach sands of Waltair coast, and its aurecology. Proc. Indian Acad. Sci. (B) 67, 201-206.

Cherbonnter, G., 1960. Complément à la faune échinodermique des Pyrenées orientales. Vie Milieu 11, 118-123.

- \& GunLe, A., 1968. Sur la présence, à Banyuls, de l'holoturie apode Labidoplax buski (M'INTosh). Bull. Mus. natn. Hist. nat., Paris (Sér. 2) 39, 1219-1223.

Clark, H., 1907. The apodous holothurians. Smithson. Contr. Washington 35, 1-231.

Flze, A., 1960. Sur un fond à Ampbioxus de la plage de Sète. Vie Milieu 11, 505-506.

Hrckman, V., 1962. Tasmanian sea-cucumbers (Holothuroidea). Pap. Proc. R. Soc. Tasm. 96, 49-72.

KöHLR, R., 1927. Les échinodermes des mers d'Europe II. Encyclopédie scientifique. Dion, Paris, $339 \mathrm{pp}$.

Monniot, F., 1962. Recherches sur les graviers à Amphioxus de la région de Banyuls-sur-Mer. Vie Milieu 13, 231-322.

Montcharmont, U., 1968. Rinvenimento di due oloturoidi (Apoda) rari, Leptosynapta minuta (Becher, 1906) e Trochodota venusta (Semon, 1887) nel Golfo di Napoli. Pubbl. Staz. zool. Napoli 36, 513-514.

Mortensen, T., 1925. Echinoderms of New Zealand and the Auckland and Campbell Islands, IV. Holothuroidea. Vidensk. Meddr dansk naturh. Foren. 79, 322-386.

Ostergren, H., 1905. Zur Kenntnis der skandinavischen und arktischen Synaptiden. Archs Zool. exp. gén. (Sér. 4) 3, 133-164.

PAwson, D., 1967. Protankyra grayi new species and Labidoplax buskii (McINTOSH) from off North Carolina (Holothuroidea; Synaptidae). Proc. biol. Soc. Wash. 80, 151-156.

- 1968. Some holothurians from Macquarie Island. Trans. R. Soc. N. Z. (Zool.) 10, 141-150.

Salvini-Plawen, L. v., 1972a. Die nordatlantische Labidoplax buski (Holothuroidea-Synaptidae) in der Adria. Zool. Anz. 188, 301-304.

- 1972b. Die Caudofoveata des Mittelmeeres und das Genus Scutopus (Mollusca, Aculifera). Proc. 5th Europ. Symp. mar. Biol. Piccin, Padova, 27-51.

Semon, R., 1887. Beiträge zur Naturgeschichte der Synaptiden des Mittelmeeres. Mitt. zool. Stn Neapel 7, 272-300.

SwEDMARK, B., 1956. Etude de la microfaune des sables marins de la région de Marseille. Archs. Zool. exp. gén. 93, 70-95.

ToRTonesE, E., 1965. Echinodermata: Fauna Ital. 6, 1-422.

Anschrift des Autors: Doz. Dr. L. v. Salvini-Plawen

I. Zoologisches Institut der Universität

Wien I

Dr.-Karl-Lueger-Ring 3

Osterreich 